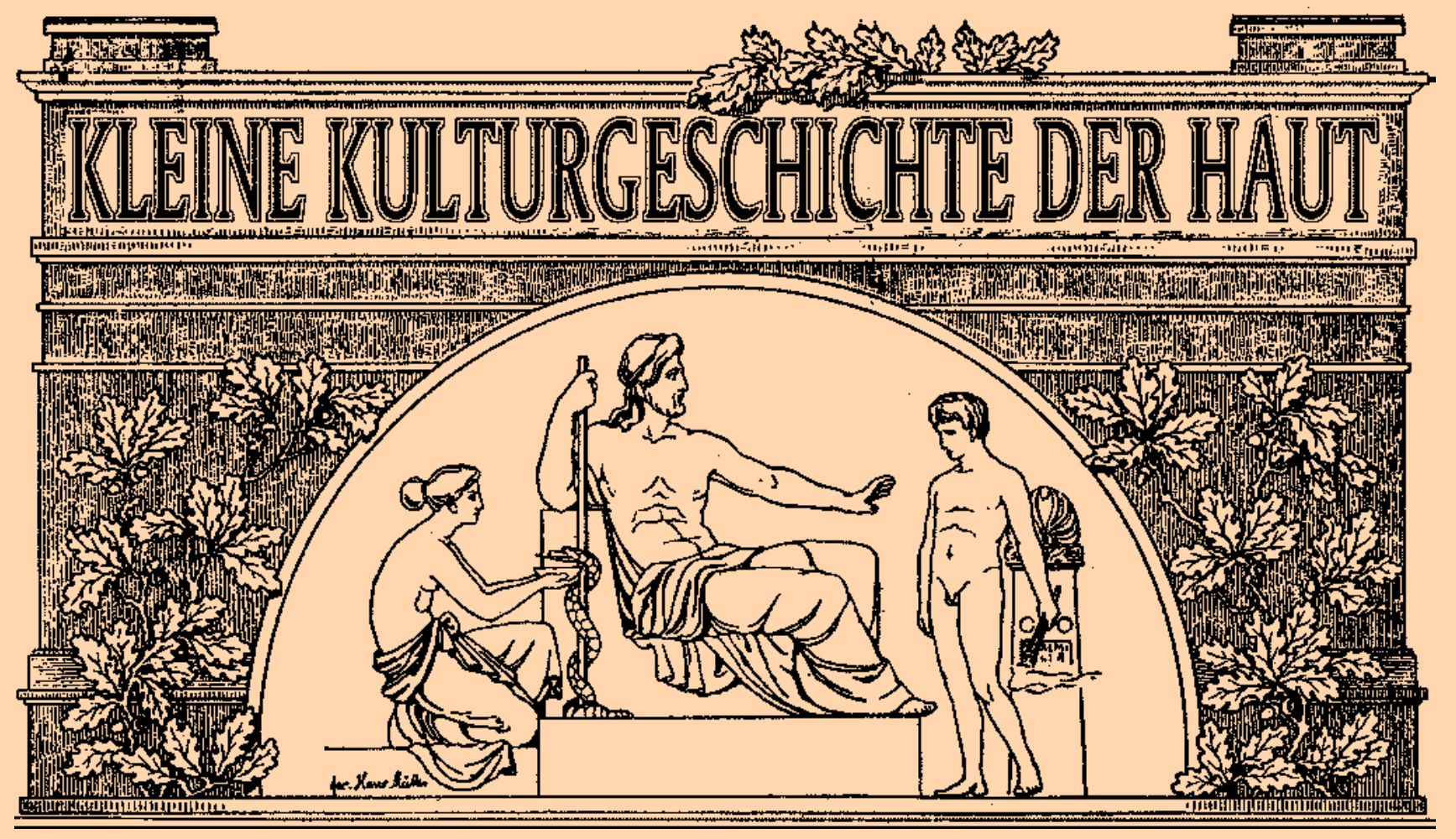

Die Zeitschrift „Aktuelle Dermatologie“ hat mit Beginn des Jahres 2004 diese Rubrik neu geöffnet, um in loser Folge kurze, prägnante und informative Artikel zu publizieren zu vielen Aspekten der Kulturgeschichte in Beziehung zur Haut. Dies erstreckt sich auf alle Kulturen und Zeiten, auf die Künste, auf Mythen und Psychologie und natürlich auch auf medizinische Aspekte.
Unsere Hoffnung, dem geneigten Leser Interessantes zu bieten, den Blick zu weiten und Freude zu bereiten, hat sich auf das Trefflichste bewährt. Reichlich Zusprüche, Kommentare, Vorschläge und aktive Teilnahme haben uns erreicht und erfreut. Dies möge weiter so gehen. Und so werden auch wir fleißig bemüht bleiben, Sie zu erbauen mit unserer kleinen Kulturgeschichte der Haut.

\title{
Weiße Indianer in Südamerika
}

\author{
White Indians in South America
}

Bibliografie

DOI $10.1055 / \mathrm{s}-2006-944777$

Akt Dermatol 2006; 32;

440-445 @ Georg Thieme

Verlag KG Stuttgart · New York

ISSN 0340-2541

Korrespondenzadresse

Dr. Manfred Reitz

Schillerstraße 7

99423 Weimar

mreitz@fli-leibniz.de
Nach der gängigen Lehrmeinung begannen erste größere Besiedlungen von Amerika vor rund 12000 bis 13000 Jahren. Damals waren die Kontinente Asien und Amerika zwischen Sibirien und Alaska durch eine gewaltige Landbrücke miteinander verbunden. Diese Landbrücke mit dem Namen Beringia war sogar während der Höhepunkte der Eiszeit eisfrei und von Menschen und Tieren besiedelt. Die Vegetation muss recht üppig gewesen sein, denn es lebten dort Großtiere, zu denen auch gewaltige Elefanten, die Mammuts, gehörten, die sich von Gras und Laub ernährten. Heute ist der Pflanzenwuchs in Nordsibirien so karg, dass auf den großen Flächen der Tundra kein Mammut mehr Nahrung finden könnte.

Der Weg nach Amerika blieb den Menschen jedoch lange verwehrt, denn auf den heutigen Ge- bieten von Alaska, Kanada und großen Teilen der USA lag ein gewaltiger und weit über 1000 Meter hoher undurchdringlicher Eispanzer. Kein Mensch konnte die riesigen Gletscherflächen überwinden, denn er wäre auf seinem Weg in den Süden verhungert. Erst als sich nach und nach eisfreie Korridore öffneten und Pflanzen sowie Tiere langsam vordrangen, konnte sich auch der Mensch auf den Weg machen. Die aus Asien kommenden Menschen gründeten deshalb erstmals vor rund 12000 Jahren in Nordamerika eine Kultur. Es war die Clovis-Kultur, benannt nach einem Ort in Neu-Mexiko, wo zuerst Werkzeuge und Waffen der Clovis-Menschen gefunden wurden.

„Clovis first“ war für lange Zeit die gesicherte Lehrmeinung zur Besiedlung von Amerika. Doch moderne Erkenntnisse können dieser These wi- 


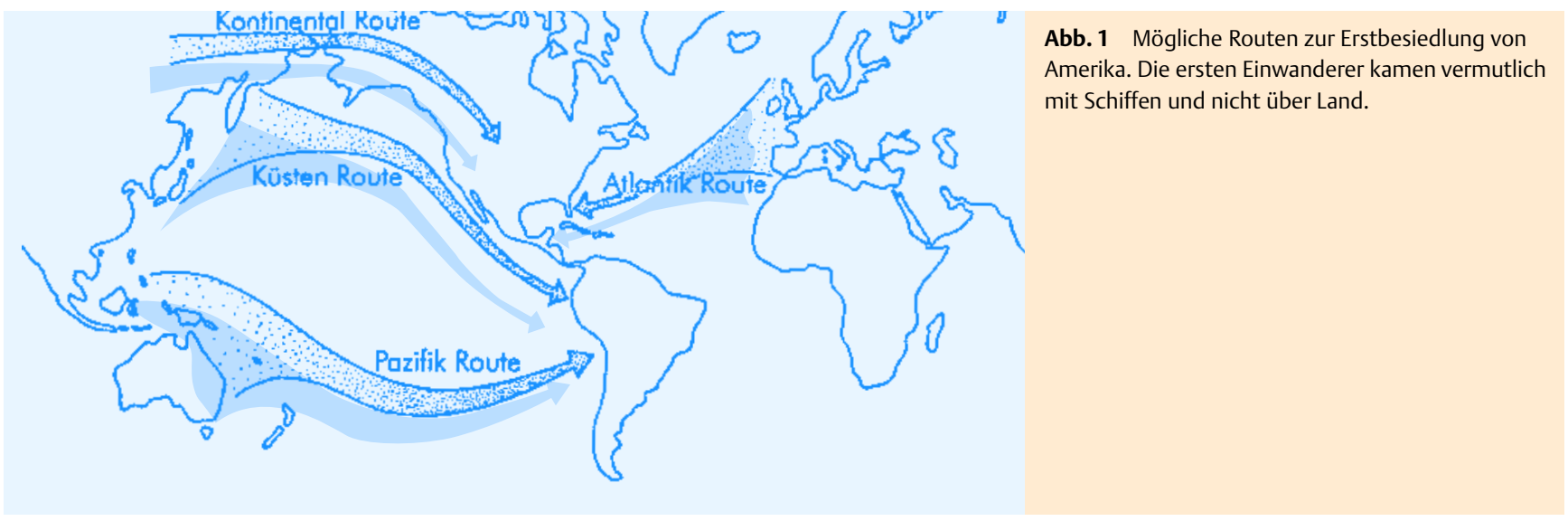

dersprechen. Der Beginn der menschlichen Besiedlung von Amerika verschiebt sich neuen Forschungsarbeiten zufolge immer weiter nach hinten. Es gab bereits lange vor der Clovis-Kultur in Amerika an unterschiedlichen Orten zahlreiche Prä-Clovis-Kulturen. Die wirklich ersten Amerikaner kamen wahrscheinlich noch nicht einmal zu Fuß über die Landbrücke von Beringia, sondern mit kleinen Booten über das Meer. Diskutiert werden mögliche Routen von Asien nach Amerika entlang der Pazifikküste, aus dem australischen Raum quer durch den Pazifik bis zur südamerikanischen Küste und von Europa aus entlang der Eisgrenze über den Atlantik bis nach Nord- und Mittelamerika ( Abb.1). Insbesondere die Route quer über den Pazifik muss ein hohes seemännisches Können erfordert haben. Es ist sogar sehr wahrscheinlich, dass sie gewählt wurde, denn in den 1970er Jahren wurde in Brasilien der Schädel von „Luzia“ gefunden, der etwa zwischen 11000 und 11500 Jahre alt ist. Vergleichende Studien verrieten eine Überraschung. Der Schädel der jungen Frau war nicht asiatisch, sondern zeigte aufgrund spezifischer Merkmale in den australisch-melanesischen Raum. Einen solchen denkbaren Kontakt haben auch die heute ausgestorbenen Ureinwohner von Feuerland hervorgehoben. Sie zeichneten sich wie „Luzia“ ebenfalls durch australisch-melanesische Merkmale aus. Wenn auch die heutigen australischen Aborigines keine Seefahrertradition mehr besitzen, ihre fernen Urahnen mussten dennoch recht tüchtige Seefahrer gewesen sein, denn sie konnten Australien nur über das Meer erreicht haben $[1,2,3]$.

\section{Südamerikanische Rätsel}

$\nabla$

Die ältesten sicheren Hinweise auf menschliche Lagerplätze oder Siedlungen stammen nicht aus Nord-, sondern aus Südamerika. Wäre Amerika ausschließlich über Beringia erschlossen worden, dann müssten Funde in Nordamerika älter sein als in Südamerika, doch es ist umgekehrt. In Chile und Brasilien, aber auch anderswo in Südamerika, gibt es Spuren von menschlichen Lagerplätzen, die rund 35000 Jahre oder älter sind und bereits auf gut organisierte Gruppen hinweisen. Manche Forscher sind sogar der Meinung, dass es in Südamerika noch weitaus ältere Hinweise auf Menschen geben könnte [2,3,4].

Die frühen Bewohner von Südamerika stammten vermutlich nur zum Teil aus Sibirien oder Beringia. Nicht nur DNA-Untersuchungen, sondern auch Zahnanalysen können hier interessante Informationen liefern. Nach Ankunft des modernen Homo sapiens in Asien entwickelten sich dort aufgrund von morphologischen Strukturänderungen bald zwei verschiedene Zahntypen, die es heute erlauben, die betroffenen Menschen in den Typ Sundadonten und Sinodonten zu unterteilen. Sundadonten lebten in „Sundaland“, eine riesige Landmasse, von der gegenwärtig nur noch die Inseln von Indonesien und den Philippinen sowie die malaiische Halbinsel vorhanden sind. Während der Eiszeit war der Meeresspiegel rund 100 Meter niedriger als heute, so dass diese zahlreichen Inseln damals Hochebenen in einer gewaltigen Landfläche waren. Vermutlich vor rund 20000 Jahren entstanden aus den Sundadonten im heutigen Nordchina und in Ostsibirien die Sinodonten, deren Zahnstruktur deutlich von der der Sundadonten abweicht. Sinodonten drangen als erste über die Landroute nach Amerika vor, während die Sundadonten erst Jahrtausende später über die Seeroute ebenfalls die Neue Welt erreichten. Die bisher ältesten Zahnfunde von Sinodonten sind in Südamerika etwa 11000 Jahre alt und stammen ausgerechnet aus dem Süden von Chile. Sie sind nur rund 1000 Jahre jünger als die vorerst ältesten menschlichen Skelettfunde in Alaska [5].

Da es während der Eiszeit in Nordamerika recht ungemütlich war, hielten sich wahrscheinlich viele Menschen nicht lange auf und wanderten bald nach Mittel- und Südamerika weiter. Von welchen Menschen die noch älteren Hinweise auf menschliche Lagerplätze in Südamerika stammen, ist weitgehend rätselhaft. Wahrscheinlich waren diese Menschen einst über das Meer gekommen. Sie verdeutlichen, Südamerika war schon früh ein Schmelztiegel der Rassen, und Zuwanderer über Land sowie über See vermischten sich. Leider wurden an vielen uralten Lagerplätzen bisher noch keine menschlichen Skelettreste gefunden $[2,3,4]$.

\section{Auf den Spuren der Pho̊nizier}

$\nabla$

Aus der Sicht der Menschen endete während der Antike die westliche Welt an der Straße von Gibraltar. Jenseits dieser Meerenge lauerten nach zahlreichen Mythen und Legenden im tobenden Atlantik nur noch unüberwindbare Gefahren. Kaum ein Seefahrer traute sich in der frühen Antike diese Grenze zu überschreiten, denn er war auf die Propaganda der Phönizier hereingefallen. Die Phönizier galten noch vor den Griechen als die besten Seefahrer der Antike. Zusätzlich waren sie tüchtige Kaufleute, die aus Furcht vor Konkurrenz alle ihre Seewege geheim hielten. Ihre Schiffe waren stabil und hochseefähig, die Besatzungen hervorragend ausgebildet ( $\bullet$ Abb. 2). Sie versorgten die Menschen des Mittelmeerraumes mit Metallen aus England, mit Waren von den Azoren und den Kanarischen Inseln sowie mit schwarzen Sklaven aus Westafrika. Im Auftrag eines ägyptischen 


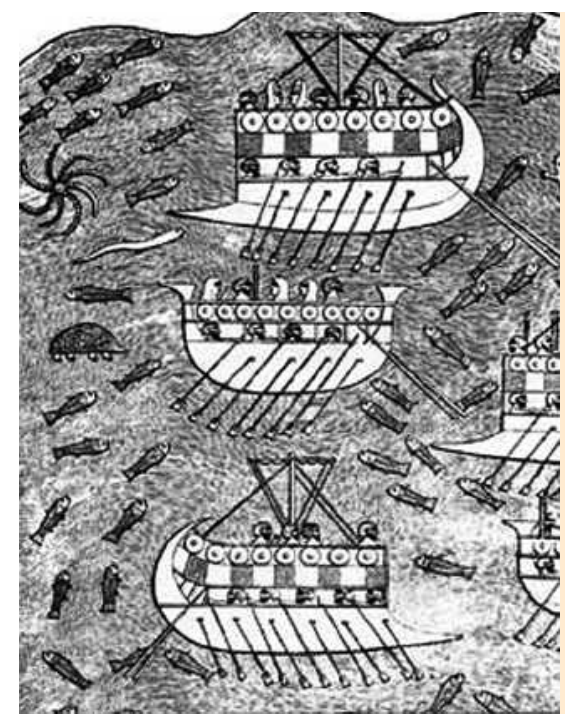

Abb. 2 Phönizische Schiffe nach einen Relief aus der Zeit um 700 v. Chr. Die Schiffe sind hochseefähig und mit Segeln sowie Ruderbänken ausgerüstet. Kriegsschiffe besitzen einen Rammsporn.

Pharaos waren sie sogar einmal um Afrika herumgesegelt. Den Atlantik betrachteten sie als ihr Meer und ließen keine fremde Kaufleute die Straße von Gibraltar passieren.

Da jenseits der Azoren Meeresströmungen direkt nach Mittelund Südamerika führen, ist es wahrscheinlich, dass phönizische Schiffe zunächst nach Stürmen zufällig nach Amerika abtrieben und dass die Neue Welt anschließend gezielt von den Phöniziern angesteuert wurde. In Brasilien wurden phönizische Inschriften und auch Amphoren gefunden, die allerdings in der Fachwelt umstritten sind. In Kunstwerken der mittelamerikanischen indianischen Hochkulturen vor Kolumbus werden manchmal Männer mit Bärten abgebildet, deren Gesichtszüge stark an Phönizier erinnern ( $\bullet$ Abb.3). Im Tempel der Krieger von Chichén Itzá in Mittelamerika sind in einer Kampfszene Menschen unterschiedlicher Rassen dargestellt. Manche Krieger besitzen eine braune Haut wie Indianer, bei anderen Kriegern ist die Haut weiß und manche von diesen haben sogar lange blonde Haare. Eine Szene dokumentiert schließlich, wie Indianer einen gefangenen weißen Krieger ihrem Gott opfern.

Die Phönizier waren Kulturvermittler, denn sie boten in der Antike vielen Mächten ihre Dienste an. Sie arbeiteten sowohl für die Herrscher von Ägypten als auch von Mesopotamien. Die Hochkultur der Olmeken in Mittelamerika, die erste aller indianischen Hochkulturen, entstand vor 1000 v. Chr. wie aus dem „Nichts“. Sie blühte plötzlich mit einem hohen Niveau auf, wobei zeitliche Parallelen zur vermuteten Ankunft der Phönizier in Amerika diskutiert werden können. Die Olmeken besaßen eine ausgereifte Bilderschrift, die Ähnlichkeiten zu den Hieroglyphen im alten Ägypten aufweist, einen komplizierten Kalender und bauten wie in Mesopotamien Stufenpyramiden. In ihrer Kunst schufen sie neben bärtigen Männern aus Ton auch riesige Köpfe aus Stein mit negroiden Gesichtszügen. Da die Phönizier häufig schwarze Sklaven als Ruderer auf ihren Schiffen mitführten, könnten diese ein Vorbild für die olmekischen Künstler gewesen sein. Bemerkenswert sind in diesem Zusammenhang Reste von Stufenpyramiden auf den Kanarischen Inseln, die angeblich von den Phöniziern gebaut wurden und möglicherweise eine Wanderung von Bauideen belegen $[2,4,6,7]$.

Während der Antike wurden die Küsten von Südamerika vermutlich sogar kartografiert. Die heute rätselhafte Karte des osmanischen Admirals Piri Reis wurde um 1513 gezeichnet ( $\bullet$ Abb.4) und zeigt deutlich die Küstenlinie von Südamerika

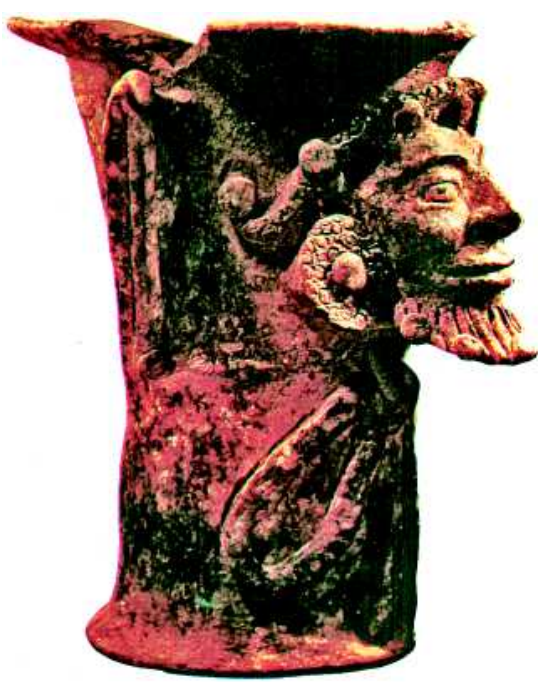

Abb. 3 Altamerikanisches Räuchergefäß aus der Zeit vor Kolumbus, gefunden in Iximche (Guatemala). Der Kopf mit Bart weist auf phönizische Gesichtszüge hin (Musée de l'homme, Paris).

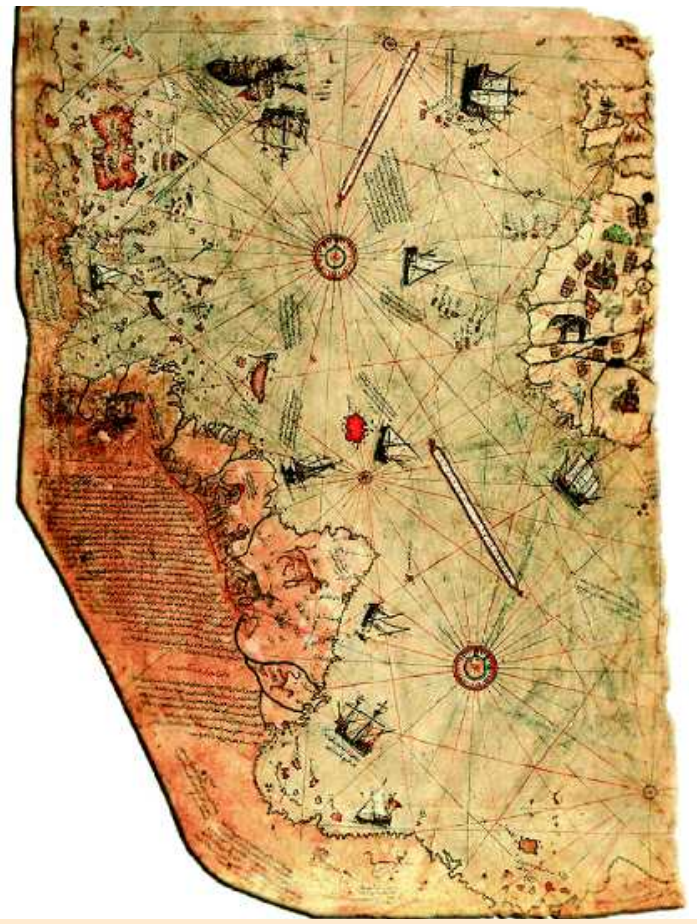

Abb. 4 Karte des Piri Reis, gezeichnet um 1513. Die Küste von Südamerika ist abgebildet. Die Quellen der Karte sollen bis in die Antike zurückgehen (Nationalbibliothek Ankara, Türkei).

einschließlich einer Landbrücke zur Antarktis, die es nur während der Eiszeit gab. Als Vorlage dienten dem Admiral nach eigenen Aussagen 20 von ihm geheim gehaltene Quellen, die bis in die Zeit von Alexander dem Großen zurückreichten.

\section{Das Schicksal der Söldner von Karthago}

Die Stadt Karthago im heutigen Tunesien war ursprünglich eine um 814 v. Chr. gegründete phönizische Kolonie gewesen und sollte den Handel im westlichen Mittelmeer sichern. Ihre Lage war strategisch sehr günstig, so dass sie sich bald selbständig machte und zu einer Großmacht heranwuchs. Karthago war lange der gefährlichste Rivale von Rom und sein genialer Feldherr 
Hannibal erschütterte das römische Selbstbewusstsein bis ins Mark. In drei gewaltigen Kriegen ging Karthago schließlich unter, und die Römer zerstörten jede Erinnerung an die einstige Großmacht. Damit verschwanden auch alle Staatsarchive und es gab keine Belege mehr, ob Karthago, wie vermutet, tatsächlich in Übersee Besitzungen unterhalten hatte. Nach dem 3. Punischen Krieg wurde Karthago zwar dem Erdboden gleich gemacht, doch große Teile der einst mächtigen Flotte und des Heeres waren noch intakt. In diesem Heer hatten einst zahlreiche keltische Söldner aus Spanien und Frankreich gekämpft, die nicht als römische Sklaven enden wollten. Es gibt Vermutungen, dass sich keltische Söldner mit ihren Familien sowie Angehörige der einstigen Oberschicht von Karthago mit den Resten der Flotte nach Südamerika abgesetzt haben. Möglicherweise fuhren sie stromaufwärts den Amazonas entlang und gründeten unterwegs immer wieder Siedlungen. Viele dieser Kelten waren blond und besaßen eine helle Haut. Sie könnten helfen, das Rätsel der blonden Indianer in Südamerika zu lösen.

Die ersten Überreste der untergegangenen Kultur der Chachapoya wurden 1843 in den östlichen Anden im Einzugsbereich der Amazonasquellen entdeckt. Die Angehörigen dieses Volkes sollen einst besonders hellhäutig, groß gewachsen sowie brünett oder blond gewesen sein und nicht wie typische Indios ausgesehen haben. Sie wohnten wie die Kelten in runden und nicht wie die indianische Bevölkerung in rechteckigen Häusern. Zu ihren Schmuckelementen an Häusern gehörten Tiere mit Hörnern, obwohl es in der vorkolumbianischen Zeit in Südamerika keine Tiere mit Hörnern gab. Bisher wurden sieben Siedlungen der Chachapoya entdeckt, darunter auch mächtige Festungen auf Berghöhen. Nach schweren Auseinandersetzungen sollen sich die kriegerischen Chachapoya erst im 15. Jahrhundert dem Reich der Inka angeschlossen haben und von diesen als gleichwertig akzeptiert worden sein. Bereits im 16. Jahrhundert ging ihre Kultur unter, hauptsächlich durch schwere von den Spaniern eingeschleppte Seuchen. Noch heute leben in ihrem ehemaligen Siedlungsgebiet hellhäutige, blonde und auch rothaarige Indios, die im Aussehen an Kelten erinnern sollen. Sie werden oft als Gringos bezeichnet, obwohl sie zu weißen US-Amerikanern in keiner Beziehung stehen $[2,3,4,6,8,9]$.

\section{Griechen, Römer und vielleicht auch Wikinger \\ $\nabla$}

Viele ehemalige Seefahrervölker traten nach den römischen Eroberungen ihrer Heimatgebiete in den Dienst des Römischen Reiches. Zu ihnen gehörten überwiegend Phönizier der Levanteküsten sowie Griechen aus den ehemaligen hellenistischen Reichen. Sie stellten den Römern fähige Kapitäne und Schiffsbesatzungen für den Fernhandel zur Verfügung und fuhren bis nach Sri Lanka sowie nach chinesischen Quellen auch nach China. Ob sie mit günstigen Meeresströmungen und Winden über den Pazifik bis nach Amerika reisten oder sogar Siedler beförderten, ist jedoch nicht belegbar. Das rätselhafte Wissen mancher antiker Historiker geht möglicherweise ebenfalls auf diese erfahrenen Seeleute zurück. Der griechische Historiker Diodorus Siculus berichtete einmal, dass die Karthager einst große Ländereien weit draußen im Atlantischen Ozean besessen hätten. Von Pausanias (um 150 n. Chr.) stammte die Behauptung, dass weit entfernt im Atlantischen Ozean auf Inseln Menschen mit roter Haut und Haaren wie schwarze Pferdeschwänze leben würden. Sogar Cicero war vermutlich informiert, denn er bemerkte einmal, weit im westlichen Ozean würde es mächtige Reiche, größer als das Römische Imperium, geben. Bemerkenswert ist schließlich auch, dass Griechen und Römer einerseits und die Maya anderseits die Farbe Purpur nach dem jeweils gleichen Rezept aus Schnecken herstellten.

In Calixtlahuaca westlich von Mexiko City wurde einmal in einem nachweislich unversehrten Grab aus der Aztekenzeit ein besonderer Fund gemacht. Es handelte sich um einen kleinen Keramikkopf, der einen bärtigen Mann zeigte und stilistisch zweifelsfrei der römischen Kunst zugeordnet werden konnte ( $\odot$ Abb.5). Physikalische Materialanalysen wiesen dem Kopf ein Alter von mindestens 1800 Jahren zu; er könnte somit in der späten Antike hergestellt worden sein. Auch wenn er im Aztekenreich produziert worden wäre, hätte der Künstler vorher genau die römische Kunst studieren müssen, so dass zumindest ein Kulturkontakt belegbar ist. Außerdem werden in Mittelamerika sowie im Süden von Nordamerika immer wieder römische Münzen gefunden, in Panama wurde sogar einmal ein original römisches Tongefäß voller authentischer Münzen $[4,9]$ entdeckt.

Der französische Forscher Jacques de Mahieu formulierte schließlich die umstrittene These, dass auch die Wikinger in Südamerika gewesen waren. Von ihren nordamerikanischen Siedlungen aus wären sie zunächst zur Halbinsel Yucatan und

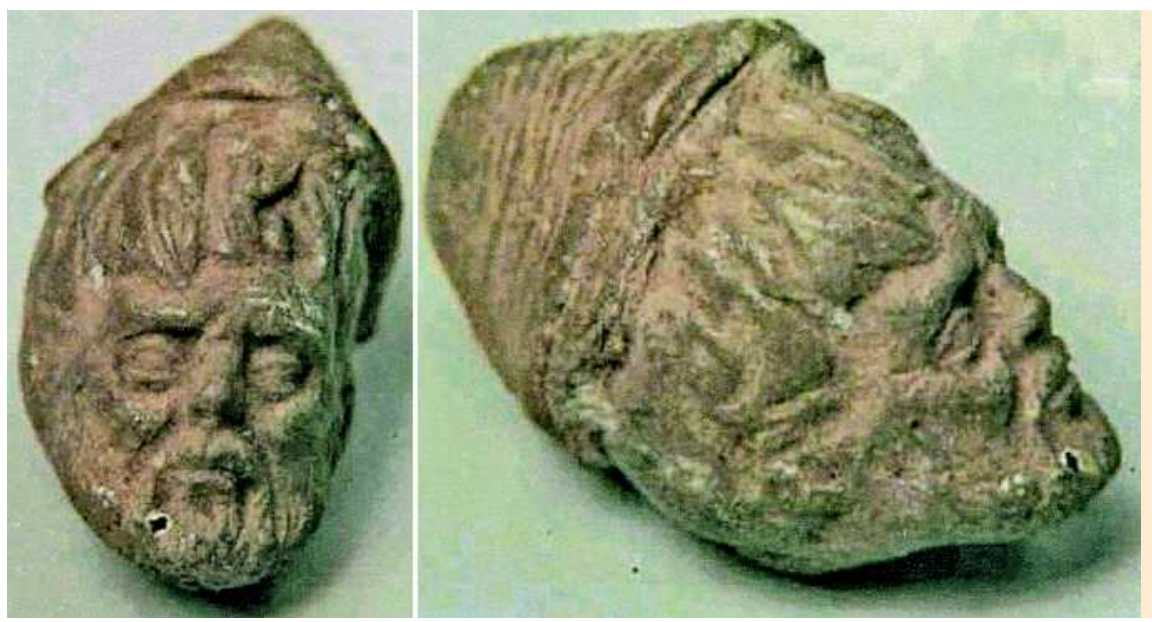

Abb. 5 Vorder- und Seitenansicht des römischen Kopfes von Calixtlahuaca, gefunden in einem intakten Aztekengrab aus der Zeit vor der Ankunft der Spanier (National-Museum für Anthropologie, Mexico-City, Mexiko). 


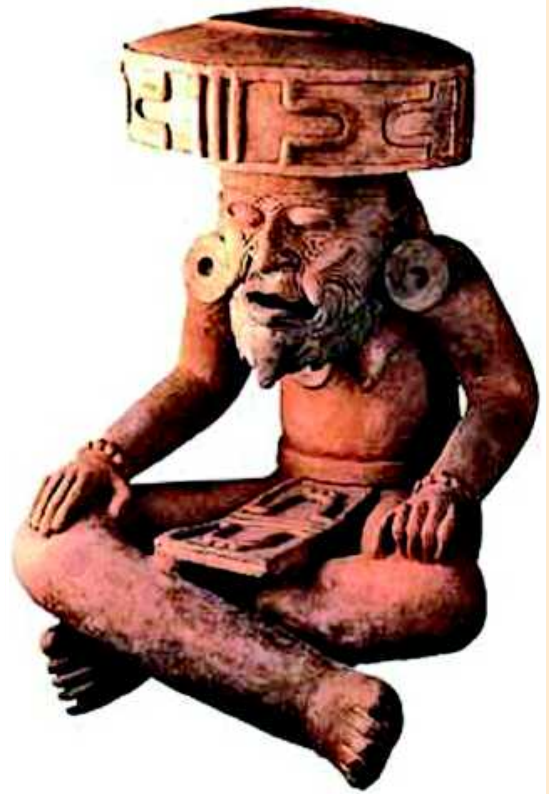

Abb. 6 Keramik von Huehueteotl, der Feuergott der Azteken. Es ist ein alter Mann mit einem Bart dargestellt, obwohl es Bartwuchs bei Indianern nicht gibt.

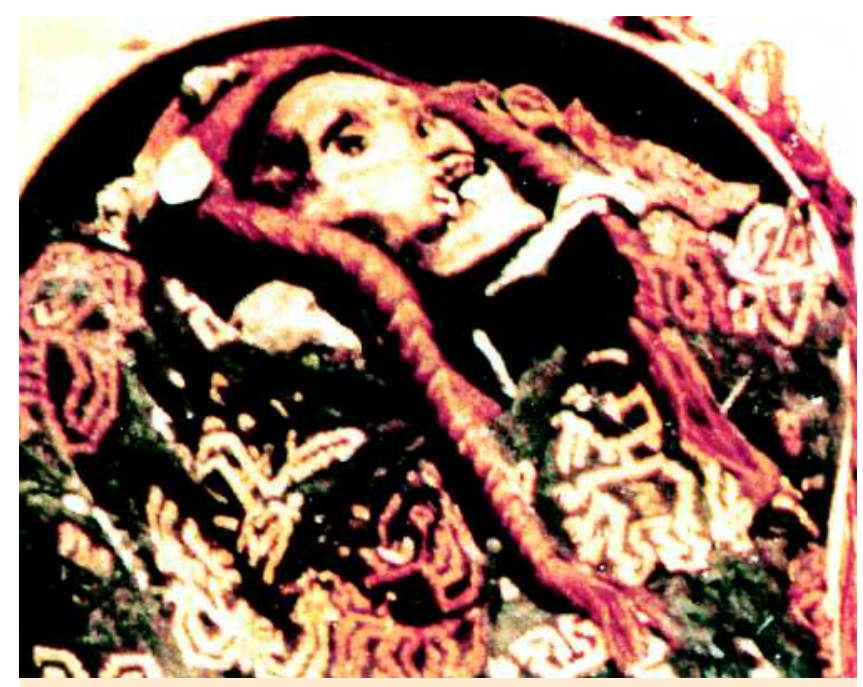

Abb. 7 Perücke aus naturblonden menschlichen Haaren auf einer Mumie der Inka-Zeit, gefunden in Paracas/Peru (National-Museum für Anthropologie, Lima, Peru).

anschließend nach Brasilien vorgedrungen. Zuletzt hätten sie sogar Südamerika umrundet und wären in Peru gelandet, wo sie mit den dortigen Kulturen Kontakte geknüpft und durch Sonnenkulte die Religionsentwicklung beeinflusst hätten. Angeblich hätte es im heutigen Paraguay vorübergehend sogar ein Wikingerreich gegeben. Zu seinen Beweisen zählte de Mahieu zahlreiche verwitterte Inschriften, die er als Runen interpretierte sowie Darstellungen von, nach seiner Meinung, Wikinger-Pferden aus vorspanischer Zeit [9].

\section{Die bärtigen weißen Götter $\nabla$}

In den Mythen der altindianischen Hochkulturen von Mexiko bis Peru wimmelt es nur so von weißen Göttern mit einem europäischen Aussehen, die einst aus weiter Ferne in das Land kamen. Huehueteotl, der Feuergott der Azteken und sowohl Vater als auch Mutter aller Götter wird als alter Mann mit einem Bart dargestellt, obwohl bei Indianern ein ausgeprägter Bartwuchs unbekannt ist ( Abb.6). Quetzalcoatl, ein Gott der Tolteken und später auch der Azteken, war nach den Mythen ein weißer, europäisch aussehender Mann, der den Menschen vermutlich um 850 n. Chr. zahlreiche Fertigkeiten lehrte und ihnen Glück brachte; er wurde als Sohn der Sonne verehrt und führte wie christliche Bischöfe einen Krummstab mit sich. Dieser bärtige weiße Gott forderte ein „christliches“ Verhalten und verachtete die bei den Azteken üblichen Menschenopfer, stattdessen opferte er den Göttern Schmetterlinge. Doch Quetzalcoatl musste mit seinen Getreuen das Land wieder verlassen, versprach jedoch seine spätere Rückkehr. Von den Maya wurde der weiße und wohltätige Gott Kukulcán verehrt, der vermutlich mit Quetzalcoatl identisch war. Die Chibcha, auch Muisca genannt, in Kolumbien verdankten nach eigenen Legenden ihr Wissen einem ebenfalls weißen bärtigen Mann mit dem Namen Bochica. Dieser Mann trug einen Stock, lange weiße Gewänder und am Hals angeblich ein Kreuz. Auch er verließ das Land und wurde durch einen anderen weißen Mann ersetzt.

Möglicherweise waren bereits im Mittelalter höchstwahrscheinlich irische Missionare in Amerika unterwegs und verbreiteten christliche Lebensformen. Der irische Mönch Brendan war angeblich um das Jahr 531 mit 12 Begleitern in Amerika gewesen. Es ist denkbar, dass er spätere Missionstätigkeiten anregte und neue Mönche schickte. Es soll sogar im Mittelalter in Amerika irische Siedlungsgebiete gegeben haben und in Florida Missionsstationen mit einer regen Reisetätigkeit der Mönche. Als der spanische Eroberer Cortez erstmals Mexiko betrat, stellten ihm Feinde der Azteken die Indianerin Malintzin als Dolmetscherin zur Verfügung. Nach ihrer Taufe wurde diese Indianerin Donna Maria genannt; jedoch ist nicht nachvollziehbar, auf welcher sprachlichen Grundlange sie sich so gut mit den Spaniern verständigen konnte.

Die Inka verehrten schließlich den weißen Heilsbringer Viracocha und seine Begleiter. Sie sollen am Titicaca-See gelebt und den Menschen ihr Wissen vermittelt haben. Aus Bewunderung hätten ihnen die Einheimischen große Bauwerke errichtet. Nach Auseinandersetzungen mussten auch Viracocha und seine Begleiter das Land wieder verlassen. Sie hätten sich im heutigen Ecuador gesammelt und wären unter Führung von Kon-Tiki hinaus in den Pazifik gesegelt. Noch heute bauen Indianer am Titicaca-See hochseefähige Schiffe aus Schilf, die allerdings nicht für das Meer bestimmt sind. Der Forscher Thor Heyerdahl unternahm mit solchen Schiffstypen sogar weite Reisen über das Meer $[2,3,4,6,8,9]$.

\section{Das Rätsel der Inka}

$\nabla$

Unter der Oberschicht der Inka soll es ungewöhnlich viele hellhäutige Menschen mit blauen Augen und manchmal sogar blonden Haaren gegeben haben. Waren Kinder des Inka-Adels blond, wurden sie „Kinder der Götter“ genannt. Pedro Pizarro, ein Vetter des Eroberers des Inka-Reiches, schwärmte in seinen Tagebüchern von den blonden und hübschen jungen Mädchen des InkaAdels. Er berichtete, Menschen gesehen zu haben, die „weißer“ waren als die Spanier. Viele Conquistadoren heirateten sogar Inka-Prinzessinen, während sie gleichzeitig die einfachen Indiofrauen als hässlich bezeichneten. Anrührend ist das Schicksal des jungen spanischen Offiziers Pedro de Barca. Er hatte beim Pokerspiel einen geraubten heiligen Spiegel der Inka aus purem 
Gold gewonnen und schenkte ihn umgehend seiner Freundin, der schönen Inka-Prinzessin Toyllor Tica, die ihn an Inka-Priester weitergab. Andere Spanier wurden nun sauer und wollten den Spiegel wieder zurück haben. Sie verfolgten das Paar und töteten es zusammen mit den Priestern am Titicaca-See. Die blonden Inka sind keine Legende. Immer wieder werden Mumien aus der Inka-Zeit mit blonden oder brünetten Haarresten gefunden. Es sind sogar Perücken aus echten naturblonden Menschenhaaren bekannt ( $\odot$ Abb. 7) $[4,6,8,9]$.

\section{Wanderwege von Kulturpflanzen \\ $\nabla$}

Die gewöhnliche Bohne Phaseolus vulgaris war bereits den Griechen und Römern bekannt und gehörte zur allgemeinen Ernährung der Menschen. Später wurde beobachtet, dass auch Indianer in Südamerika die gleiche Bohnenart wie die Europäer kultivierten. Lange Zeit wurde angenommen, dass die Spanier einst die Bohne in die Neue Welt eingeführt und die Indianer sie als Nahrungsmittel übernommen hatten. Doch Grabfunde in Peru machten diese Vorstellungen zunichte. In einem unversehrten Grab aus der Zeit vor den Inka fanden Forscher in Mittelperu Bohnen als Totennahrung. Sie waren dem Toten als Proviant für das Jenseits mitgegeben worden und waren mit den Bohnen der Griechen und Römer identisch. Bohnen wurden zuerst in der Alten Welt kultiviert und in Amerika gibt es keine Wildformen von ihnen. Ihr Weg in die Neue Welt ist rätselhaft.

Baumwolle birgt als Beleg für frühe weltweite Handelsbeziehungen und Atlantiküberquerungen ebenfalls noch manches Geheimnis. In Mesopotamien und Ägypten wurde schon früh Baumwolle angebaut, deren Zellen 13 große Chromosomen besitzen. In Amerika gibt es eine wild wachsende Baumwollart mit 13 kleinen Chromosomen, deren Fäden allerdings für eine Textilproduktion nicht geeignet sind. Dennoch haben Baumwollstoffe in den altamerikanischen Hochkulturen eine lange Tradition, und die Inka waren Meister der Webkunst. Von Mexiko bis Peru bauten die Indianer lange vor Kolumbus eine Baumwollart zur Textilherstellung an, deren Zellen 13 kleine und 13 große Chromosomen enthielten. Es handelte sich um eine Kreuzung, einen Hybriden, zwischen der kultivierten orientalischen Baumwollart und der wilden amerikanischen Baumwollart. Nach Meinung von Botanikern konnte eine solche Kreuzung nicht zufällig entstanden sein, sondern wurde von Menschen durchgeführt [6].

\section{Literatur}

1 Scarre C (ed). The Human Past. London, New York: Thames \& Hudson; 2005

2 Lavallée D. The First South Americans: The Peopling of a Continent from the Earliest Evidence to High Culture. Salt Lake City: Univ. Utah Press; 2000

3 Bruhns KO. Ancient South America. Cambridge, New York: Cambridge Univ. Press; 1994

4 Oth R. Bevor Kolumbus kam. Die frühen Entdecker Amerikas. Stuttgart: Theiss Verlag; 2006

5 Turner CG. Dentrochronical separation estimates for pacific rim populations. Sciene 1986; 232: 1140 - 1142

6 Schoch RM, McNally RA. Die Weltreisen der Pyramidenbauer. Frankfurt: Zweitausendeins; 2002

7 Diehl R. The Olmecs: America's First Civilization. London, New York: Thames \& Hudson; 2004

8 Moseley ME. The Incas and Their Ancestors. The Archaeology of Peru. London, New York: Thames \& Hudson; 2001

9 Zillmer H-J. Kolumbus kam als Letzter. München: Langen Müller; 2004

\section{Leserbrief}

Schirren C, Jung EG

Die Universitäre Dermatologie in Kiel und die Dermatologen-

familie Schirren: Fruchtbare Wechselbeziehungen

Akt Dermatol; 32: 265-267

Mit Interesse habe ich den Beitrag von C. Schirren und E. G. Jung über die fruchtbare Entwicklung der Kieler Dermatologie in Bezug auf die Praxis der Familie Schirren und die UniversitätsHautklinik gelesen. Es ist unbestritten, dass eine Historie, wie sie die Familie Schirren in der Dermatologie aufweist, einzigartig ist und besondere Beachtung verdient. Durch zahlreiche in verschiedensten Medien publizierte Beiträge vor allem von C. Schirren selbst wird immer wieder auf diese besondere Rolle hingewiesen.

Spricht man von einer befruchtenden Entwicklung zwischen zwei Bereichen, ist anzunehmen, dass beide Bereiche, also auch die Universitäts-Hautklinik Kiel, zu dieser Befruchtung beigetragen haben. Kaum ein anderer der Kieler Klinikdirektoren hat die Hautklinik so entscheidend geprägt wie mein Vorgänger Prof. Dr. med. Dr. h.c. Enno Christophers. Ich empfinde es daher als bedauerlich, dass Prof. Christophers in dem Artikel kein einziges Mal außer in der tabellarischen Aufstellung der Klinikdirektoren erwähnt wird. In den fast 30 Jahren, in denen Prof. Christophers die Kieler Hautklinik geleitet hat, ist der Aufbruch von der traditionellen, beschreibenden Dermatologie zu einem wissenschaftlich hoch qualifizierten Fach vollzogen worden. Prof. Christophers war einer der Pioniere, die der Grundlagenforschung durch Anstellung von Naturwissenschaftlern den entsprechenden Stellenwert innerhalb einer Klinik einräumten. Die bahnbrechenden Arbeiten von Prof. Dr. rer. nat. Jens-Michael Schröder, der weltweit höchste Anerkennung auf dem Gebiet der natürlichen Immunität der Haut besitzt, bezeugen dies. Prof. Christophers hat neue organisatorische Strukturen wie die Tagesklinik (die erste in der deutschen Dermatologie) eingeführt und der Kieler Dermatologie internationales Ansehen verschafft. Wenn diese von Schirren und Jung dargestellte Wechselwirkung in der Tat so fruchtbar gewesen ist, ist dies auf der akademischen Seite sicher der Person von Prof. Christophers zu verdanken, da eine positive Wechselwirkung mit der Hautklinik ohne ihn in seiner Ära nicht denkbar und auch nicht möglich gewesen wäre. Es freut mich, dass die geschätzten Autoren mir als derzeitigem Klinikdirektor Zukunftsfähigkeit attestieren. Diesem Attribut fühle ich mich auch gewachsen, vor allem aber aufgrund der Tatsache, dass mir Prof. Christophers ein die langen Jahre exzellent geführtes Haus mit unter seiner Leitung hervorragend ausgebildeten, hoch motivierten und vor allem extrem loyalen Mitarbeitern übergeben hat, wofür ich ihm ewig dankbar sein werde.

Prof. Dr. med. Thomas Schwarz

Direktor der Universitäts-Hautklinik Kiel

Schittenhelmstraße 7, 24105 Kiel

E-mail: tschwarz@dermatology.uni-kiel.de 